\title{
Case Report: - Aortic Valve Replacement due to Aortic Valve Leaflet Perforation after PCI
}

\author{
* Yasser Shaban Mubarak, MD. **Muhammad Hussian Abdel Wahaab, MD. \\ *Assistant Professor of Cardiothoracic Surgery Department, Faculty of Medicine, Minia University, Egypt. \\ **Consultant adult cardiac surgery at Madinah Cardiac Center, King Fahad Hospital, Madinah, KSA.
}

\begin{abstract}
Percutaneous Coronary Intervention (PCI) is widely recognized as an effective treatment for Acute Coronary Syndrome (ACS). Inspite of advances in equipment and experience of interventional cardiologist, still there are rare complications occurred [1].

Iatrogenic injury of the aortic valve leaflet is a rare. Aortic insufficiency (AI) after a PCI suggests an iatrogenic valve injury. Aortic leaflet injury is not common but possible complication of PCI. Because of the serious consequences, it should be mentioned in the informed consent. Aortic repair of iatrogenic injury is possible, and it can be performed with excellent clinical and functional midterm results. So, Aortic valve replacement (AVR) is the last option [2].
\end{abstract}

Keywords:- Aortic Leaflet Perforation, Traumatic Aortic Regurgitation, Valve Injury, Percutaneous Coronary Intervention, Aortic Valve Replacement.

\section{INTRODUCTION}

PCI is a safe technique that has a good result with ischemic heart disease (IHD). However, despite its advantages, it is not risk-free. Acute traumatic (AI) due to catheterization injury is an extremely rare mechanical complication. The mechanism of acute traumatic (AI): (a) transitory regurgitation due to movement of rigid catheters into the aorta arch, and the condition is relieved upon catheter withdrawal (b) irreversible acute AI is extremely rare and is related to stents implanted in the RCA ostium protruding into the aorta (c) direct catheter perforation of aortic valve leaflet, during intervention in the RCA with Amplatz catheters. This type of complication can be prevented by careful catheter handling, especially in situations where catheterization is challenging or in patients with coronary anomalies [3].

\section{CASE REPORT}

Male patient 55 years old known as smoker, hypertensive, diabetic and dyslipidemic presented to our hospital with Non-ST segment Elevation Myocardial Infarction (Non-STEMI). His past history recorded multiple admissions with acute coronary syndrome (ACS) and two PCIs (Stents to Diagonal and RCA in 2015, then Proximal LAD and $1^{\text {st }}$ obtuse marginal in 2019). Transthoracic echocardiogram (TTE) was showed preserved biventricular systolic function without any valvular lesion. Emergency coronary angiogram was performed, due to ongoing chest pain refractory to medical treatment and recent PCI, through radial artery and showed significant distal LM ( Left main),calcified angulated lesion in LAD/CX (Left anterior descending and circumflex), with RCA instent restenosis. He refused CABG and accepted high risk PCI. Complex PCI was done by multiple trials to engaged left coronary ostia, which failed after multiple trial, and wire was failed to pass through RCA lesion. During the procedure he developed acute heart failure due to recent infarction and arrhythmia. Cardiac markers were elevated before PCI but more elevated Post-PCI failure. Postprocedure TTE showed moderately severe AI with probable aortic valve injury and $\mathrm{EF}=35 \%$. After stabilization at $\mathrm{CCU}$ (Coronary Care Unit), trans-esophageal echocardiogram (TEE) was done and showed tear in the NCC with jet of AI (figures 1\&2). We received emergency call from CCU for $\mathrm{CABG}$ and aortic intervention. Emergency surgery was performed by establishing CPB (Cardio-Pulmonary Bypass) and on pump harvesting LIMA (left internal mammary artery). Then, distal anastomosis of SVG (saphenous vein graft) to RCA, and OM were done. Then, aortotomy was performed, inspection of aortic leaflets revealed tear in NCC and excluded endocarditis (figure 3). Aortic valve replacement (AVR) was performed because of extensive cusp tear was beyond repair. Also, the AV repair needs more experience and takes more time which is not suitable in that instability condition. After that, LIMA to LAD and proximal SVG anastomosis were done. IABP (Intra-Aortic Balloon Pump) was inserted with moderate inotropic support was added for easily weaning. Postoperative course was passed uneventful by expert CSICU (Cardiac Surgery Intensive Care Unit).Follow up echo is revealed well function prosthetic aortic valve without gradient, and improvement of EF ( $45 \%$ ) and biventricular function after 6 months.

\section{DISCUSSION}

AI caused by a leaflet perforation is seen most frequently with infective endocarditis (IE) involving aortic valve [4]. Iatrogenic aortic valve injury and leaflet perforation have been reported after cardiac operations performed such as repair of a bicuspid aortic valve, aortic valve decalcification, and septal myectomy [7]. Rarely, suture-related, inadvertent injury to an aortic valve leaflet can produce leaflet tear with regurgitation after cardiac operations performed in the vicinity of the aortic valve (e.g., mitral valve replacement, repair of a membranous ventricular septal defect, and repair of an ostium primum atrial septal defect) [8]. But in our case, previous cardiac 
surgery and IE was excluded by TEE, Lab., and intraoperative exploration.

Iatrogenic AI is caused by multiple stalling of rotabur in calcified coronary artery caused traction and disengagement of guide causing rotabur to be freely hanged in ascending aorta close to cusps. Very high speed rotablator come in contact with aortic leaflet causing perforation and tear [9]. In our case, rotablator was not used.

Although rare post PCI complications (AI, Aortic Dissection, Coronary Artery Dissection); the incidence and clinical implications of these warrants further investigation given the high mortality rate and the growing number of cardiac procedures performed [1]. So, we try to collect all post PCI complications, those need surgical intervention.

AI can be caused temporary during Impella implantation due to direct contact with aortic leaflet or permanent after removal due to aortic leaflet injury [5]. In our case, there is not any device used other than PCI.

In urgent CABG, LIMA can be safely harvested and hemodynamic instability is no longer a relative contraindication. In standard CABG, LIMA is harvested before establishing CPB. Patients with severe multi-vessel $\mathrm{CAD}$ and tight Left main stem stenosis can become unstable during induction of anesthesia or during surgery. Routine clinical practice in these patients is to establish urgent cardiopulmonary bypass, and only vein conduit is used to bypass all significant coronaries which are easy and rapid to harvest in such condition. So, these patients are deprived of LIMA graft and its long term patency [6]. So, we harvested LIMA after establishing CPB for safety of the patient and because of instability of hemodynamics without deprived patient from long term patency graft to LV (Left Ventricle) and relative young patient.

We performed LIMA on LAD and SVG on OM, which are previously stented because coronary angiography was showed significant distal LM stenosis.

So, any cardiac intervention needs surgical backup team who trained well and has experience to deal with rare but lifesaving conditions.

\section{CONCLUSION}

Iatrogenic AI is very rare complication of PCI. Aortic valve repair if leaflets tears or perforations are repairable, otherwise, AVR with complete revascularization is safe procedure.

\section{$>$ Abbreviations:}

PCI: Percutaneous Coronary Intervention, ACS: Acute Coronary Syndrome, AI: Aortic Insufficiency, AVR:
Aortic Valve Replacement, IHD: Ischemic Heart Disease, RCA: Right Coronary Artery, LAD: Left Anterior Descending, LCX: Left Circumflex, OM: Obtuse Marginal, SVG: Saphenous Vein Graft, Non-STEMI: non ST segment elevated Myocardial Infarction, TTE: Trans Thoracic Echo., TEE: Trans Esophageal Echo., IE: Infective Endocarditis. CCU: Coronary Care Unit. LIMA: Left Internal Mammary Artery. IABP: Intra-Aortic Balloon Pump. CPB: Cardio-Pulmonary Bypass. CSICU: Cardiac Surgery Intensive Care Unit. LV: Left Ventricle.

\section{REFERENCES}

[1]. Lau C, McRoy G, Mahendraraj K, Kulkarni K, Chamberlain R. Aortic Dissection after Percutaneous Coronary Intervention for Acute Coronary Syndrome: An Outcomes-Based Study from the Nationwide Inpatient Sample Database. Intern J Clinic Med. 2017, 8, 21-33.

[2]. Fitsum L, Paul P. Aortic Valve Leaflet Perforation after Minimally Invasive Mitral Valve Repair. Ann Thorac Surg. 2016; 101:1180 - 82.

[3]. Sergio G, Santiago J, Camacho $F$ et al. Percutaneous Coronary Intervention: A Rare Cause of Acute Traumatic Aortic Regurgitation. Rev Esp Cardiol. 2017; 70(6):501-14.

[4]. Ballal RS, Mahan EF, Nanda NC. Aortic and mitral valve perforation: diagnosis by trans-esophageal echocardiography and Doppler color flow imaging. Am Heart J. 1991; 121:214- 17.

[5]. Nishimura K, Iida M, Shimokawa T. Prosthetic valve replacement for aortic leaflet tear secondary to Impella device placement. Journal of Artificial Organs. https://doi.org/10.1007/s10047-020-01191-y.

[6]. Sher-i-Murtaza M, Ahmad M. On pump harvesting of Left Internal Mammary Artery (LIMA) in unstable patients undergoing coronary artery bypass grafting (CABG) is a safe operative strategy: A pilot study. Pak J Med Sci. 2019; 35(3): 605-608.

[7]. Freeman WK, Schaff HV, Orszulak TA, Tajik AJ. Ultrasonic aortic valve decalcification: serial Doppler echocardiographic follow-up. J Am Coll Cardiol. 1990; 16: 623-30.

[8]. Arthur C, Ramesh C, Anees J, Meizhen L, Leonard L, Steven R. Echocardiographic Recognition of Iatrogenic Aortic Valve Leaflet Perforation. Ann Thorac Surg. 1997; 64:684-89.

[9]. Vadgaonkar V, Tarief H, Shivappa S, Sulaibikh L, Saif S, Alnuwakhtha A. P249 Iatrogenic aortic cusp laceration causing severe acute traumatic aortic regurgitation secondary to complex percutaneous coronary intervention using rotablator; 3DTEEanatomic correlates. Eur H J Cardiovasc Imaging.2020;

21(1). https://doi.org/10.1093/ehjci/jez319.111 


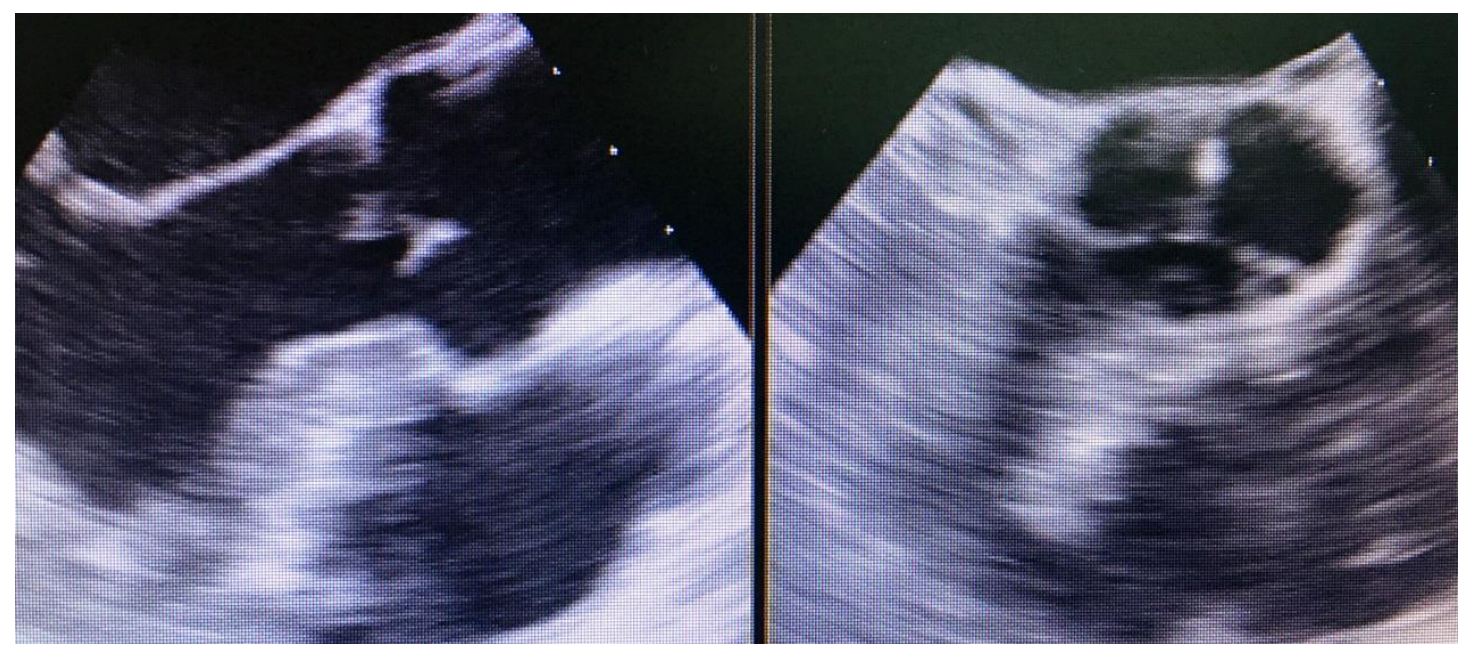

Fig 1:- TEE is showing NCC tear in AV leaflet.

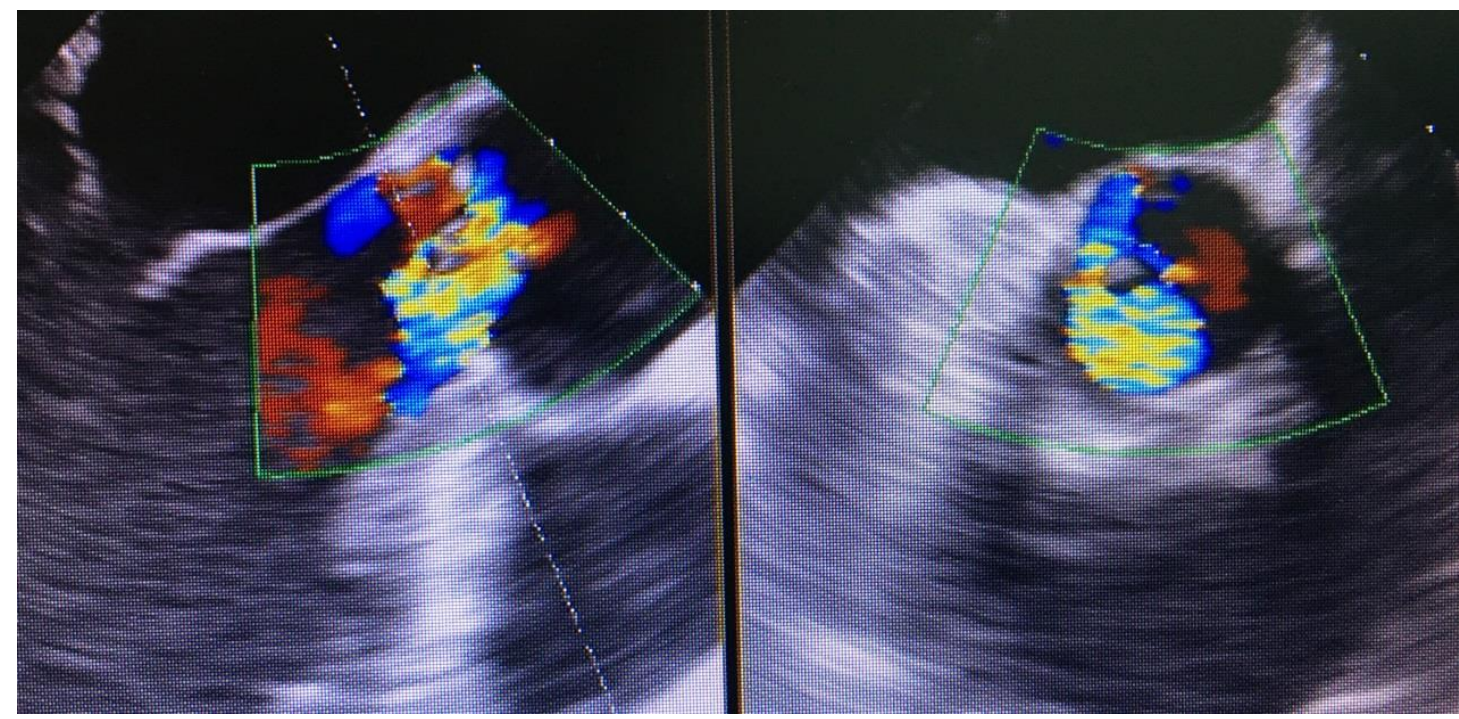

Fig 2:- TEE is showing severe AR, eccentric jet related to NCC

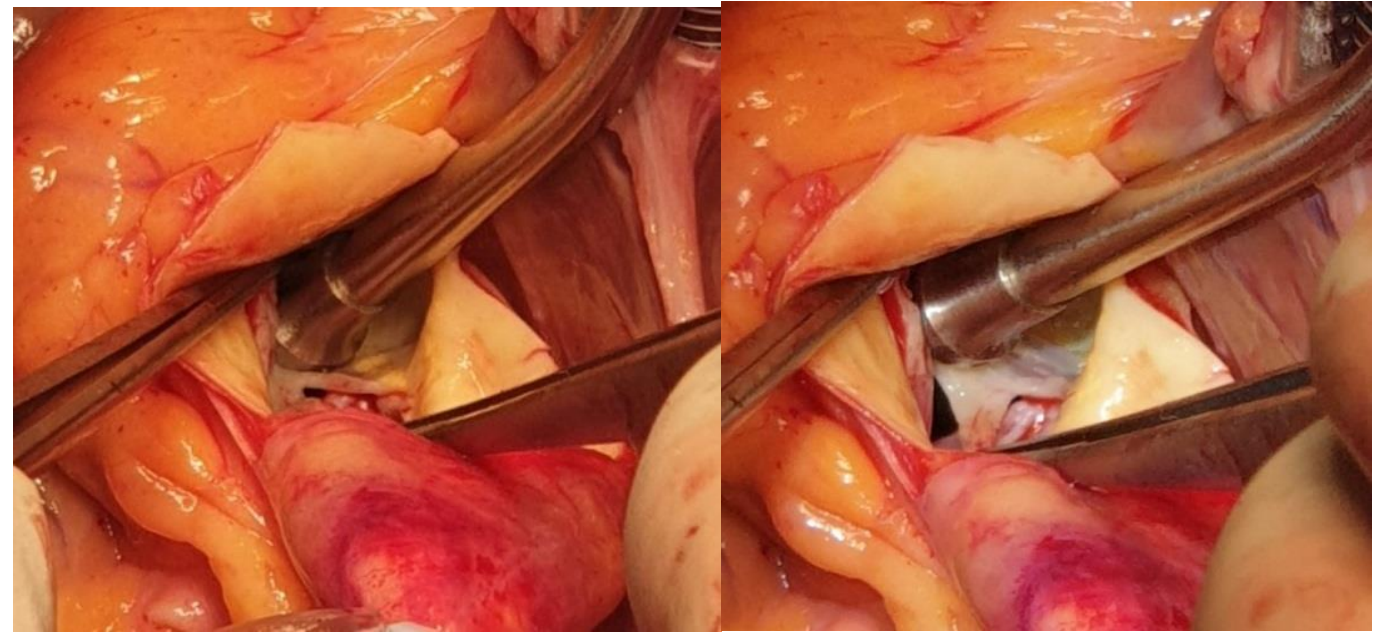

Fig 3:- Intraoperative view is showing tear in NCC. 